\title{
Pheromone Static Routing Strategy for Complex Networks
}

\author{
Xiang Ling ${ }^{1}$, Henry Y.K. Lau ${ }^{2}$, Rui Jiang ${ }^{1}$, and Mao-Bin Hu* \\ 1.School of Engineering Science, \\ University of Science and Technology of China, \\ Hefei 230026, People's Republic of China \\ 2.Department of Industrial and Manufacturing Systems Engineering, \\ The University of Hong Kong, \\ Pokfulam Road, Hong Kong, P.R. China
}

(Dated: November 7, 2018)

\begin{abstract}
In this paper, we adopt the concept of pheromone to generate a set of static paths that can reach the performance of global dynamic routing strategy [Phys. Rev. E 81, 016113(2010)]. In the test stage, pheromone is dropped to the nodes by packets forwarded by the global dynamic routing strategy. After that, static paths are generated according to the density of pheromone. The output paths can greatly improve traffic systems' overall capacity on different network structures, including scale-free networks, small-world networks and random graphs. Because the paths are static, the system needs much less computational resource than the global dynamic routing strategy.

PACS numbers: 89.75.Hc, 45.70.Vn, 05.70.Fh
\end{abstract}

*Electronic address: humaobin@ustc.edu.cn 


\section{INTRODUCTION}

Traffic on networked systems is important for many modern communication and transportation systems. Since the discovery of Small-World phenomenon [1], and Scale-Free property [2], it is widely proved that the topology and degree distribution of networks have profound effects on the processes taking place on these networks, including traffic flow [3-11].

Routing is the control mechanism that directs each commuter in a networked system from its source to destination through a serial of switching nodes. A good routing strategy can improve network traffic efficiency without changing the underlying infrastructures \1216]. Therefore, adopting efficient routing strategies are often preferred by the engineers. In modern communication and transportation systems, the shortest path protocol is often used. However, the shortest paths often lead to the collapse of hub nodes, which greatly reduces system performance. To avoid this problem, some routing strategies are proposed. One good example is the efficient routing strategy [13]. The efficient path between any pair of nodes is defined as the path that the sum degree of nodes is minimum, denoted as:

$$
\text { Path }=\min \sum_{m=1}^{l} k\left(x_{m}\right)^{\beta},
$$

where $k\left(x_{m}\right)$ is the degree of $m$ th node along the path, $l$ is the path length and $\beta$ is a tunable parameter. When $\beta=1$, Eq.(1) can reach a very high network capacity, which is almost ten times larger than shortest paths. Actually, the efficient routing strategy propels the packets to use the low-degree nodes, which composes the peripheral of the network. Therefore, the capacity of low-degree nodes can be utilized and so the system's capacity can be improved.

The nodes' ability can not be fully reflected by the degree. The best routing strategy to fully utilize the nodes' ability might be accomplished by examine the queue length of nodes. Recently, Ling etal proposed a global dynamic routing strategy [16], in which the path between any pair of nodes is defined as the sum of nodes' queue length is minimum:

$$
\text { Path }=\min \sum_{m=1}^{l}\left[1+n\left(x_{m}\right)\right]
$$

where $n\left(x_{m}\right)$ is the queue length of node $x_{m}$. This routing strategy selects the path along which the nodes have least traffic loads. Thus the network capacity can be further enhanced to almost double of the efficient routing strategy. As far as we know, the global dynamic routing strategy can achieve the highest performance for scale-free networks. Nevertheless, 
the shortcoming of this routing protocol lies in its high computational consumption since the paths are dynamical. This problem can be acute when the network size is large. An approach to reach the performance of global dynamic routing strategy with static paths is needed.

In this brief report, we propose that by the help of "pheromone", this kind of static routing paths can be obtained. The concept of "pheromone" has been proved to be efficient to form a good local routing strategy [15]. Here it is demonstrated that "pheromone" can also help to generate routing paths that can reach high traffic capacities as the global dynamic routing strategy for scale-free, small-world and random network infrastructures.

\section{PHEROMONE STATIC ROUTING STRATEGY}

In the process of moving, ants will release a special kind of secretion pheromone, which helps other ants to look for paths [17, 18]. The pheromone released by the pioneer packets are important for the final paths to be established. Similarly, we suppose that some pioneer packets travel in the network by the global dynamic paths and drop an information of "pheromone" to the nodes in the first period. Therefore, the traffic flow characters of global dynamic routing strategy can be reserved. Then the static routing paths are generated by the "pheromone" information. Finally, the packets are forwarded with the output routing paths. The paths are static and the packets do not need to drop "pheromone" any more.

The traffic model is described as follows. Initially, the pheromone density of each node is set to zero. The maximal queue length of each node is assumed to be unlimited and First-In-Fist-Out (FIFO) discipline is applied. The nodes' delivering ability is set to $C=1$, i.e., each node can forward only one packet to its neighboring nodes in each step.

(1) Test Stage: In this stage with preset length of $T_{t}$ steps, $N_{p t}$ test packets are forwarded in the system by the global dynamic routing strategy [16]. The routing paths are update in every step. Meanwhile, each test packet waiting in the queue will release one unit of pheromone to the current node for each step. That is, if a test packet waits for 5 steps in the queue before being delivered, the pheromone density of this node will increase by 5 units. The unit pheromone value is set to $\delta p=0.0001$. When a test packet arrives at its destination, it remains in the system and is forwarded to another random destination. After $T_{t}$ steps, the nodes in the system will have different pheromone densities and the test 
packets are all removed.

(2) Path Generation Stage: The pheromone static path for any pair of nodes is generated that the sum pheromone density of nodes along the path is minimum:

$$
\text { Path }=\min \sum_{m=1}^{l} p\left(x_{m}\right),
$$

where $p\left(x_{m}\right)$ is the pheromone density of node $x_{m}$.

(3) Delivery Stage: In each step, $R$ packets enter the system with randomly chosen sources and destinations. The packets are forwarded by the pheromone static paths. The paths do not need to be updated. Once a packet arrives at its destination, it is removed from the system. Following common practice, we adopt the order parameter [4]:

$$
\eta(R)=\lim _{t \rightarrow \infty} \frac{C}{R} \frac{\left\langle\Delta N_{p}\right\rangle}{\Delta t}
$$

where $\Delta N_{p}=N_{p}(t+\Delta t)-N_{p}(t), N_{p}(t)$ is the number of packets within the network at time $t$, and $\langle\ldots\rangle$ indicates the average over time windows of width $\Delta t$. With increasing packet generation rate $R$, there will be a critical value of $R_{c}$ which characterizes the phase transition from free flow $(\eta=0)$ to congestion $(\eta>0)$. In the free flow state, $\eta$ is around zero due to the balance of created and removed packets. When $R$ exceeds $R_{c}$, the packets accumulate in the network, and $\eta$ becomes positive. The network's overall capacity can be measured by the maximal generating rate $R_{c}$. $R_{c}$ can also be estimated analytically by the maximal node betweenness in the network [5]:

$$
R_{c}=\frac{N(N-1)}{B_{\max }}
$$

where betweenness $(B)$ is defined as the number of paths passing through a node, and $N$ is the network size.

We note that this model is different from the ant colony algorithm [17, 18] because it does not consider the evaporation effect of pheromone and the pheromone only appears in the first two stages. Actually, the concept of pheromone is borrowed only to record the traffic flow trace of global dynamic routing strategy. From the above model, one can also see that the model can be adjusted by changing the parameters of testing time length $T_{t}$ and test packet number $N_{p t}$. In the following, we will show the results on three typical network structures: the Barabási-Albert (BA) scale-free networks [2], the Newman-Watts small-world networks [19] and the Erdös-Rényi (ER) random graphs [20]. 


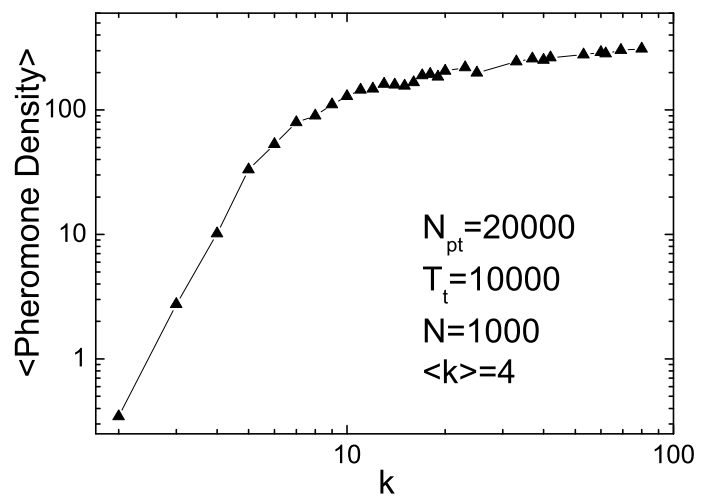

FIG. 1: Pheromone density vs node degree $k$ with test packet number $N_{p t}=20000$ and testing time $T_{t}=10000$. Network parameters are size $N=1000$ and average degree $\langle k\rangle=4$.

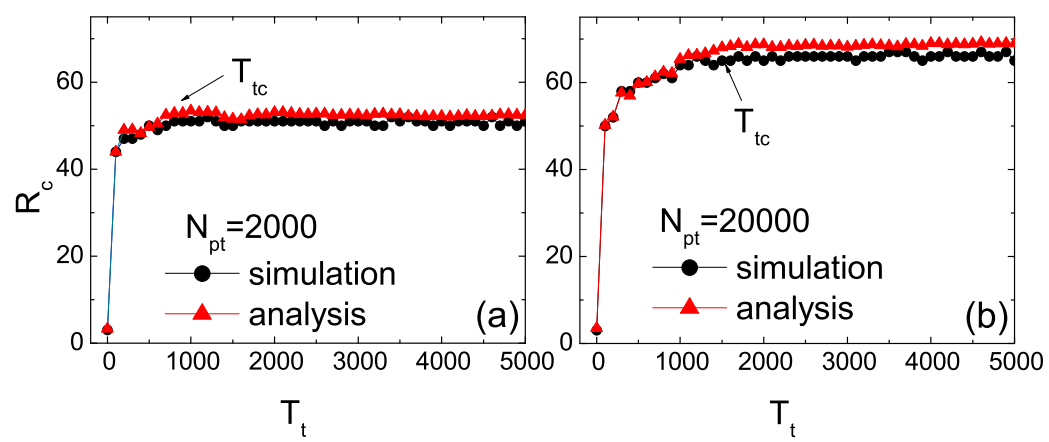

FIG. 2: (Color online.) Variation of system capacity $R_{c}$ with the length of test period $T_{t}$ with $N_{p t}=2000$ (a) and $N_{p t}=20000$ (b). Each data point is obtained by carry out the model on one BA network realization with parameters $N=1000$ and $\langle k\rangle=4$.

\section{SIMULATION AND ANALYTICAL RESULTS}

We firstly present the simulation and analytical results on BA scale-free networks [2]. In this model, starting from $m_{0}$ fully connected nodes, a new node with $m$ links is added to the existing graph at each step according to the preferential attachment. The probability for the new node to be connected to an existing node $i$ is proportional to the degree $k_{i}$ of the node. 

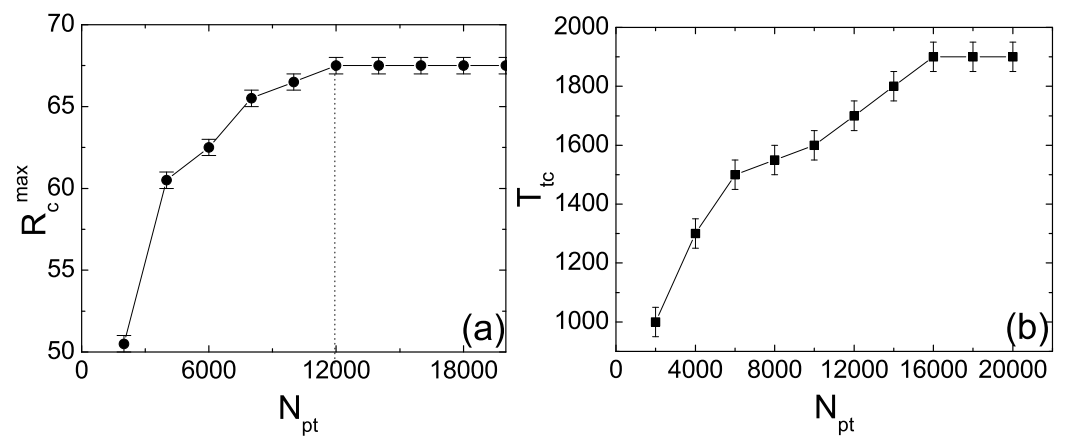

FIG. 3: (a) Maximal capacity $R_{c}^{\max }$ vs $N_{p t}$ with $T_{t}=10000$. (b) Saturation point $T_{t c}$ vs $N_{p t}$ for the system. Network parameters are $N=1000$ and $\langle k\rangle=4$.
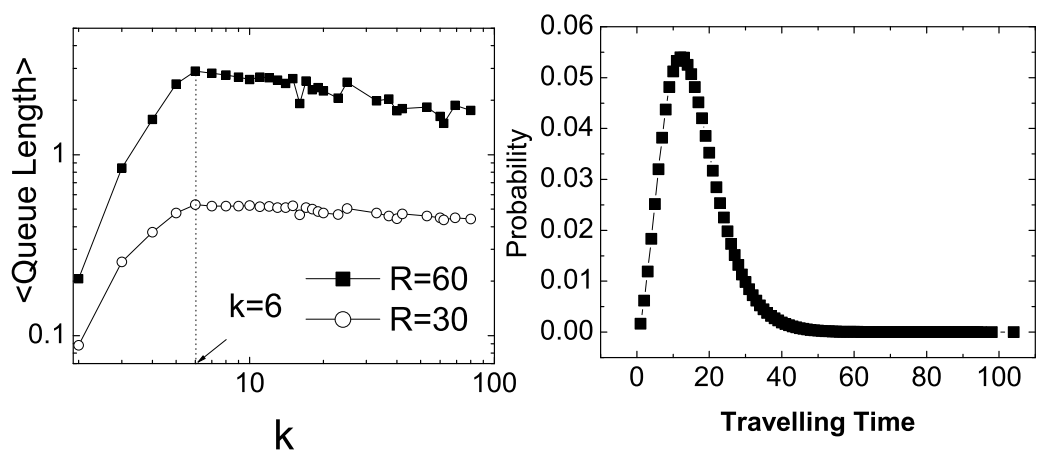

FIG. 4: (a) Average queue length vs node degree $k$ with the pheromone static routing strategy in free flow state. (b) The probability distribution of travelling time in free flow state $(R=60)$. Network parameters are $N=1000$ and $\langle k\rangle=4$.

Figure 1 shows a typical distribution of pheromone as function of node degree at the end of test stage. It approximately follows a piecewise power law with positive exponent. This indicates that the hub nodes are more burdened with the global dynamic routing strategy.

Figure 2 shows the results of network traffic capacity measured by $R_{c}$ with changing $T_{t}$. The analytical estimation of $R_{c}$ by Eq.(5) are shown with Betweenness calculated by the pheromone static paths. For a given $N_{p t}$, network capacity $R_{c}$ increases with $T_{t}$ until it reaches saturation at a critical point of $T_{t c}$. The saturated network capacity $R_{c}^{\max }$ is affected by $N_{p t}$. When $N_{p t}=12000$, the maximum network capacity is $R_{c}^{\max }=68$. This is very 


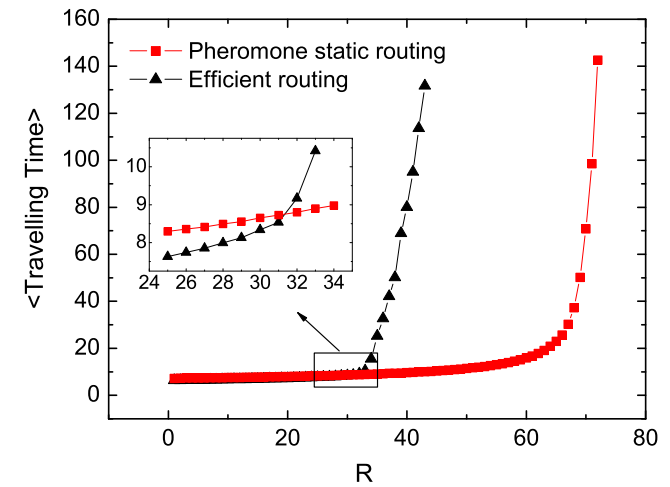

FIG. 5: (Color online). Average travelling time for different $R$ with network size $N=1000$ and average degree $\langle k\rangle=4$. When $R>R_{c}$, the average travelling time is calculated by averaging the travelling time of arriving packets during 1000 steps of delivery.

close to that of the global dynamic routing strategy $\left(R_{c}=71\right)$ [16] and is more than double of efficient routing strategy $\left(R_{c}=33\right)$ [13] with the same network parameters. One can see that the analytical results are slightly larger than simulation results. This discrepancy might be due to the noise in the simulation.

In Fig $3(\mathrm{a})$, one can see that $R_{c}^{\max }$ increases with $N_{p t}$ and come to a saturate value of 68 at $N_{p t}=12000$. In Fig!3(b), we also show the variation of critical point $T_{t c}$ for different $N_{p t}$.

Figure 4(a) shows the distribution of average queue length as function of node degree $k$ with the pheromone static paths. For both cases $R=60<R_{c}$ and $R=30<R_{c}$, one can see that the traffic load distributes non-linearly with a maximum at $k=6$. This indicates that the traffic load on hub nodes is alleviated. This behavior is different from the situations of shortest path and global dynamic routing strategy where the queue length follows a power law of $n(k) \sim k^{\gamma}$ with exponent $\gamma>0$. Figure 4(b) shows the probability distribution of packet's travelling time in free flow state $\left(R=60<R_{c}\right)$ with the pheromone static paths. The travelling time is recorded as the time that the packet spend from source to destination. It approximately follows a Poisson distribution, which is similar to that of global dynamic routing strategy [16].

Figure 5 shows the variation of packets' average travelling time over packet generating rate $R$ for the efficient routing strategy and pheromone static paths. one can see that the 

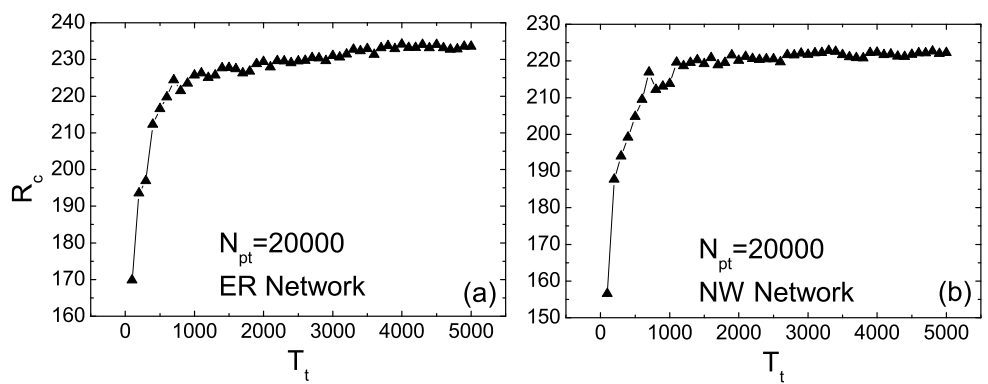

FIG. 6: Analytical estimation of network traffic capacity $R_{c}$ vs testing time $T_{t}$ with $N_{p t}=20000$ for: (a) Erdos-Renyi random networks of $N=1000$ and $\langle k\rangle=8$; (b) Newman-Watts small-world networks of $N=1000$ and $\langle k\rangle=8$.

average travelling time is slightly larger than that with efficient routing strategy for $R<32$. When $R>32$, the system enters congestion for efficient routing strategy, thus the packet's travelling time increases rapidly. When $R>68$, the system is also congested with pheromone static paths, thus the average travelling time also increases.

Finally we present the results of network traffic capacity with Newman-Watts (NW) small-world networks [19] and Erdös-Rényi (ER) random graphs [20]. NW small-world networks are generated by randomly adding long-range links to a one-dimensional lattice with nearest and next-nearest interactions with periodic boundary conditions [19]. ER random graphs are generated by connecting couples of randomly selected nodes, prohibiting multiple connections [20]. We find the model can also achieve much higher system capacity on these networks. In Fig [6, the analytical results of network traffic capacity measured by $R_{c}$ are shown for the two type of networks. The overall capacity can reach a maximum of $R_{c}^{\max } \approx 234$ for ER networks and $R_{c}^{\max } \approx 222$ for NW networks. For comparison, we find by simulation that $R_{c} \approx 50$ with shortest path routing strategy and $R_{c} \approx 140$ with efficient routing strategy for ER networks with the same parameters. For NW networks, $R_{c} \approx 85$ with shortest path routing strategy and $R_{c} \approx 155$ with efficient routing strategy. 


\section{SUMMARY}

In summary, a set of static routing paths is proposed for the traffic system on complex network infrastructures. Remarkably, the paths can almost reach the high performance of global dynamic routing strategy with a fixed routing table. It is demonstrated that the model can perform well on different network structures, include scale-free, small-world and random networks. For scale-free networks, the distribution of queue length and probability of travelling time are also investigated. This model can help the plan and design of routing protocols of modern communication and transportation networks.

\section{Acknowledgments}

This work is funded by the National Natural Science Foundation of China with Nos.10872194, 11072239, the Fundamental Research Funds for the Central Universities of China. M.-B. Hu acknowledges the support of Massey University International Visitor Research Fund and the Endeavour Australia Cheung Kong Research Fellowship.

[1] D.J. Watts and S. H. Strogaz, Nature (London) 393, 440 (1998).

[2] A.-L. Barabási, R. Albert, Science 286, 509 (1999).

[3] S. Boccaletti, V. Latora, Y. Moreno, Phys. Rep. 424, 175 (2006).

[4] A. Arenas, A. Díaz-Guilera, R. Guimerá, Phys. Rev. Lett. 86, 3196 (2001).

[5] R. Guimerà, A. Díaz-Guilera, F. Vega-Redondo, A. Cabrales, A. Arenas, Phys. Rev. Lett 89, $248701(2002)$.

[6] B. Tadić, S. Thurner, G. J. Rodgers, Phys. Rev. E 69, 036102 (2004).

[7] P. Echenique, J. Gómez-Gardeñes, Y. Moreno, EPL 71, 325 (2005).

[8] H. Zhang, Z.H. Liu, M. Tang, P.M. Hui, Phys. Lett. A 364, 177 (2007).

[9] J. Zhang, X. B. Cao, W. B. Du, K. Q. Cai, Physica A 389, 3922 (2010).

[10] C. L. Chen, X. B. Cao, W. B. Du, Physica A 389, 4571 (2010).

[11] X. B. Cao, W. B. Du, C. L. Chen, J. Zhang, Chin. Phys. Lett. 28, 058902 (2011).

[12] J.M. Kleinberg, Nature(London) 406, 845 (2000).

[13] G. Yan, T. Zhou, B. Hu, Z.Q. Fu, B.H. Wang, Phys. Rev. E 73, 046108 (2006). 
[14] W.X. Wang, B.H. Wang, C.Y. Yin, Y.B. Xie, T. Zhou, Phys. Rev. E 73, 026111 (2006).

[15] X. Ling, M.B. Hu, R. Jiang, R.L. Wang, X.B. Cao, Q.S. Wu, Phys. Rev. E 80, 066110 (2009).

[16] X. Ling, M.B. Hu, R. Jiang, Q.S. Wu, Phys. Rev. E 81, 016113 (2010).

[17] A. Colorni, M. Dorigo, V. Maniezzo et al, Proceedings of the 1st European Conference on Artificial Life 134 (1991).

[18] E. Bonabeau, M. Dorigo, G. Theraulaz, Nature(London) 406, 39 (2009).

[19] M.E.J. Newman, D.J. Watts, Phys. Rev. E 60, 7332 (1999).

[20] P. Erdös, A. Rényi, Publ. Math. Debrecen 6, 290 (1959). 